\title{
SIRT7 Regulates the Vascular Smooth Muscle Cells Proliferation and Migration via Wnt/ $\beta$-Catenin Signaling Pathway
}

\author{
Jianghua Zheng, ${ }^{1}$ Kai Chen, ${ }^{1}$ Haifei Wang, ${ }^{1}$ Zhilong Chen, ${ }^{1}$ Yong Xi, ${ }^{1}$ Hongshun Yin, ${ }^{1}$ \\ Kun Lai, ${ }^{1}$ and Yujuan Liu $\mathbb{D}^{2}$ \\ ${ }^{1}$ Department of Vascular Surgery, Affiliated Hospital of North Sichuan Medical College, China \\ ${ }^{2}$ Department of Gynaecology and Obstetrics, Affiliated Hospital of North Sichuan Medical College, China \\ Correspondence should be addressed to Yujuan Liu; liuyujuan996@163.com
}

Received 21 September 2018; Revised 9 November 2018; Accepted 15 November 2018; Published 4 December 2018

Academic Editor: Mitomu Kioi

Copyright (C) 2018 Jianghua Zheng et al. This is an open access article distributed under the Creative Commons Attribution License, which permits unrestricted use, distribution, and reproduction in any medium, provided the original work is properly cited.

\begin{abstract}
A huge amount of evidence indicates that sirtuin 7 (SIRT7), a key mediator of many cellular activities, plays a crucial role in the pathogenesis of various diseases. However, little is known about the role of SIRT7 in atherosclerosis. This study investigated the potential role of SIRT7 in regulating the proliferation and migration of human vascular smooth muscle cells (HAVSMCs) and its possible molecular mechanism. In this study, human vascular smooth muscle cells (HAVSMCs) were induced by oxidized lowdensity lipoprotein (ox-LDL) to establish atherosclerosis (AS) cell model. Immunofluorescence staining and Western blot were used to detect the level of $\alpha$-SMA expression, which was a marker protein in AS. In addition, RT-qPCR and Western blot assay were applied for exploring the mRNA and protein expression levels of SIRT7, Wnt, $\beta$-catenin, and cyclin D1 after knockdown or overexpression of SIRT7. And, furthermore, Cell Counting Kit-8 assay, flow cytometry, and wound-healing assay were used to assess HAVSMCs proliferation, cell cycle, and migration. Dickkopf-1 (DKK-1), a secretory glycoprotein that can block Wnt/ $\beta$ catenin pathway, was used in SIRT7 overexpression HAVSMCs; subsequently cells proliferation and migration were assessed by Cell Counting Kit-8 assay, flow cytometry analysis, and wound-healing assay. We found that knockdown of SIRT7 significantly promoted cell proliferation and migration, decreased the percentages of cells in the G1 and G2 phases, and increased those in the $S$ phase and downregulated the protein expression levels of Wnt, $\beta$-catenin, and cyclin D1, while overexpression of SIRT7 had reverse results. After treatment with Wnt/beta-catenin pathway inhibitor DKK-1 in SIRT7 overexpression HAVSMCs, cell proliferation and migration were increased, respectively. In conclusion, SIRT7 inhibited HAVSMCs proliferation and migration via enhancing $\mathrm{Wnt} / \beta$-catenin activation, which provided a novel therapeutic strategy for antiatherosclerosis.
\end{abstract}

\section{Introduction}

Atherosclerosis (AS) is a chronic degenerative disease of the arterial wall, which is well known for its high morbidity and mortality in aged people around the world [1]. A growing body of evidences have demonstrated that abnormal proliferation and migration of vascular smooth muscle cells (VSMCs) play a significant role in AS progression $[2,3]$. Accumulating reports suggest that oxidized lowdensity lipoprotein (ox-LDL), a key risk atherogenic factor in the development progression of AS, contributes to VSMCs proliferation and migration $[4,5]$. Therefore, we choose oxLDL-stimulated VSMCs to induce AS cell model in order to explore the relative regulatory mechanisms.
The sirtuins (SIRTs) protein family belongs to class III of histone deacetylases and consists of seven members (denoted SIRT1-7). Increasing amounts of evidence indicate that SIRTs can regulate plenty of cellular processes, such as stress responses, metabolism, cell cycle, inflammation, and senescence as well as apoptosis [6]. Furthermore, SIRTs also participate and have diverse roles in cardiovascular, neurodegenerative diseases and cancer [7]. SIRT1, the most widely studied mammalian sirtuin, could suppress CD40 expression via NF-kB pathway in human umbilical vein endothelial cell [8]. It has been well documented that SIRT1 protects against DNA damage and inhibits atherosclerosis in VSMCs [9]. In addition, impaired SIRT1 promotes the migration of vascular smooth muscle cell-derived foam cells [10]. SIRT7, the latest 
characterized SIRT, is the youngest member in SIRTs protein family, and several compelling breakthrough reports show that SITR7 plays a decisive role in cell proliferation, migration, survival, and protein synthesis as an important cellular regulator [11-13]. The previous studies reported that SIRT7 inhibited proliferation and migration in several cancer cells $[14,15]$. SIRT7 as a tumor suppressor plays an important role in suppressing the epithelial-to-mesenchymal transition in oral squamous cell carcinoma [16]. Moreover, the expression of SIRT7 was downregulated in thyroid carcinoma and lower SIRT7 levels were observed in big tumor size [17]. However, the role of SIRT7 in AS remains poorly elucidated.

A recent study shows that SIRT7 is instrumental for osteogenic differentiation of bone marrow stem cells, partly by activation of the $\mathrm{Wnt} / \beta$-catenin signaling pathway [18]. Accumulating evidence shows that $\mathrm{Wnt} / \beta$-catenin signaling exerts a critical role in vascular pathologies. For example, several studies suggest that the $\beta$-catenin/T-cell factor (TCF) signaling pathway can regulate VSMCs proliferation and vascular calcification $[19,20]$. In addition, evidence is emerging to indicate that $\mathrm{Wnt} / \beta$-catenin signaling participates in regulating cellular proliferation, inflammation, and cell deposition fate, which are important causes in AS progression [21-23]. All of these studies arouse our interests in whether SIRT7 and $\mathrm{Wnt} / \beta$-catenin signaling play roles in resisting AS and the underlying regulatory relationship between them. Therefore, Dickkopf1 (DKK-1), a secretory glycoprotein that can block the Wnt/ $\beta$-catenin pathway by competitively binding to receptors on the cell membrane, was used in our present study [24].

In this study, we demonstrated that the expression of SIRT7 was downregulated in HAVSMCs stimulated by oxLDL. Moreover, SIRT7 knockdown enhances HAVSMCs proliferation and migration accompanied with decreasing protein expression levels of Wnt, $\beta$-catenin, and cyclin D1, while SIRT7 overexpression had opposite results in parallel studies. The protein expression levels of Wnt, $\beta$-catenin, and cyclin D1 were decreased after DKK-1 blocking Wnt/betacatenin pathway. In conclusion, SIRT7 inhibited VSMCs proliferation and migration via activating $\mathrm{Wnt} / \beta$-catenin.

\section{Materials and Methods}

2.1. Cell Culture and Exposure to ox-LDL. The human aortic vascular smooth muscle cells T/G HAVSMCs (HAVSMCs) were obtained from China Center for Type Culture Collection (Wuhan, China) and maintained in DMEM medium (Gibco, USA) supplemented with $10 \%$ fetal bovine serum (FBS, Gibco, USA), $100 \mu \mathrm{g} / \mathrm{ml}$ penicillin, and $100 \mu \mathrm{g} / \mathrm{ml}$ streptomycin at $37^{\circ} \mathrm{C}$ in a $5 \% \mathrm{CO}_{2}$ humidified incubator. HAVSMCs were seeded in 6-well plates at the density of $1 \times$ $10^{5}$ cells/well. Subsequently, they were exposed to $100 \mu \mathrm{g} / \mathrm{ml}$ ox-LDL (Yiyuan Biotech, Guangzhou, China) for $24 \mathrm{~h}$ to establish the AS cell model. Cells with passage number between 10 and 15 were used for the experiments.

2.2. Cell Transfection. HAVSMCs were seeded in 6-well plates at the density of $1 \times 10^{6}$ cells/well. When grown at $40-60 \%$ confluence, HAVSMCs were transfected with $100 \mathrm{nM}$ of
SIRT7 siRNA or control siRNA in Opti-MEM with Lipofectamine RNAiMAX (Invitrogen, Carlsbad, CA). SIRT7 siRNA (siSIRT7-1: $\quad 5^{\prime}$-CCUGCCGUGUGAGGCGGAA-3' and siSIRT7-2: $5^{\prime}$-GCCGUGUGAGGCGGAAGCG-3') and control siRNA (siControl: $5^{\prime}$-CCUUGCGUGGGAGCGCGAA$3^{\prime}$ ) were synthesized by Invitrogen (Carlsbad, CA, USA). The overexpression of SIRT7 was made with the pcDNA3.1 plasmid (BioVector, Beijing, China) which was performed using Lipofectamine 2000 (Invitrogen, Carlsbad, CA). Then successful transfections were determined by RT-qPCR and Western blot assay after cells were incubated for $24 \mathrm{~h}$.

\subsection{Immunofluorescence Staining and Analysis. HAVSMCs} were seeded in 24-well plates which contained coverslips. After different treatment, cells were fixed with $4 \%$ paraformaldehyde for $15 \mathrm{~min}$. Then $0.1 \%$ Triton X-100 in TBS was used to permeabilize the fixed cells for $20 \mathrm{~min}$ at room temperature. After being blocked in 5\% bovine serum albumin for $30 \mathrm{~min}$, cells were incubated with anti$\alpha$-SMA (Santa Cruz Biotechnology, CA, USA) overnight at $4^{\circ} \mathrm{C}$. Following washing three times with PBS, cells were incubated with DyLight ${ }^{\mathrm{TM}}$ 488-conjugated secondary antibodies (Thermo Scientific) for $1 \mathrm{~h}$ at room temperature. Then the nuclei were subsequently stained with $4^{\prime}$,6-diamidino-2phenylindole (DAPI; Sigma-Aldrich; 1:1000) for $5 \mathrm{~min}$ and then washed three times with PBS in the dark. Immunofluorescence was detected using a confocal laser scanning microscope (Olympus FV1000).

2.4. Cell Proliferation Assay. To explore the effect of SIRT7 knockdown or SIRT7 overexpression on the proliferation of ox-LDL-induced HAVSMCs, cells were seeded in 96-well plates at a density of $5 \times 10^{3}$ cells/well (three replications per group) and incubated at $37^{\circ} \mathrm{C}$ for $6 \mathrm{~h}$. The cell viability was examined by CCK-8 kit (Dojindo Laboratories, Kumamoto, Japan) according to the manufacturer's instruction. At the indicated time points $(24,48$, and $72 \mathrm{~h}), 10 \mu \mathrm{lCCK}-8$ solution was added to each well. Following incubation at $37^{\circ} \mathrm{C}$ for $1 \mathrm{~h}$, the absorbance was measured with a plate reader at $450 \mathrm{~nm}$.

2.5. Cell Migration Assay. For the wound-healing assay, cells were plated in 12-well plates at the density of $1 \times 10^{5}$ cells/well (three replications per group). When cells were grown at $80 \%$ confluence and merged into a monolayer, a sterile $200 \mu \mathrm{l}$ pipette tip was used to gently generate scratch wounds at the surface of the cell layer. Then cells were rinsed twice with serum-free media to remove cell debris. An inverted microscope (BX51; Olympus Corporation; 20X magnification) was used to monitor cells at the borders of the scratches. The degree of scratch healing was observed and images were captured in each group at 0 and $24 \mathrm{~h}$.

2.6. Flow Cytometry. The cell cycle analysis was performed using the cell cycle analysis kit (Beyotime Biotechnology, Jiangsu, China). Following transfection for $48 \mathrm{~h}, 1$ $\times 10^{5}$ cells were treated with $70 \%$ cold ethanol at $4^{\circ} \mathrm{C}$ overnight to increase cell membrane penetrability. Subsequently, $100 \mu \mathrm{g} / \mathrm{ml}$ RNase (Keygentec, Nanjing, China) was 
used to treat cells at $37^{\circ} \mathrm{C}$ for $20 \mathrm{~min}$. Following staining with $30 \mu \mathrm{g} / \mathrm{ml}$ propidium iodide (Keygentec, Nanjing, China) for $30 \mathrm{~min}$ at room temperature in the dark, cell cycle was analyzed by Gallios Flow Cytometry (Beckman Coulter, USA). The flow cytometry results were evaluated using a Cell Quest kit (BD Biosciences, CA, USA).

2.7. Quantitative Reverse Transcription-PCR (RT-qPCR). Total RNA was extracted using the TRIzol Reagent (Invitrogen, Carlsbad, CA, USA). RNA was reversetranscribed to cDNA using the QuantiTect RT kit (Qiagen) following the manufacturer's instructions. Real-time PCR was performed using a System 7500 instrument (Applied Biosystems, Carlsbad, CA, USA) and the following PCR cycling protocol was used: $95^{\circ} \mathrm{C}$ for $5 \mathrm{~min}$ and then 40 cycles of $94^{\circ} \mathrm{C}$ for $15 \mathrm{~s}, 55^{\circ} \mathrm{C}$ for $20 \mathrm{~s}$, and $72^{\circ} \mathrm{C}$ for 20 $\mathrm{s}$, followed by $72^{\circ} \mathrm{C}$ for $7 \mathrm{~min}$. The mRNA expression level of GAPDH was the endogenous control. The primer sequences for qPCR were as follows: SIRT7, forward $5^{\prime}$-ACGCCAAATACTTGGTCGTCT-3' and reverse $5^{\prime}$ AGCACTAACGCTTCTCCCTTT-3'; GAPDH, forward $5^{\prime}$-CTCACCGGATGCACCAATGTT- $3^{\prime}$ and reverse $5^{\prime}$ CGCGTTGCTCACAATGTTCAT- $3^{\prime}$. Results were analyzed following the $2^{-\Delta \Delta \mathrm{Cq}}$ calculation method [25].

2.8. Western Blotting. VSMCs proteins were extracted with RIPA lysis buffer (Beyotime, China) and incubated for $30 \mathrm{~min}$ on ice. Then the supernatants were collected after centrifugation. The concentrations were detected using a BCA protein assay kit (Bio-Rad, China). Equal amounts of proteins were loaded onto $10 \%$ SDS-polyacrylamide gels to separate various proteins which were transferred to PVDF membranes subsequently. Then these membranes were blocked with a buffer containing 10\% non-fat milk in PBS for $2 \mathrm{~h}$ at room temperature, followed by incubation with primary antibodies overnight at $4^{\circ} \mathrm{C}$, respectively. Then the membranes were incubated with the goat anti-rabbit horseradish peroxidaseconjugated IgG secondary antibodies (1:1,000; cat. no. A0208; Beyotime Institute of Biotechnology, Haimen, China) at room temperature for $1 \mathrm{~h}$. Following washing three times with TBST, signals were detected using the enhanced chemiluminescence reagent (Millipore, Billerica, MA, USA) and the ImageJ software was provided to analyze the fold changes of protein levels. Anti- $\alpha$-SMA (1:1000; cat. no. 19245S), antiSIRT7 (1:1000; cat. no. 5360S), anti- $\beta$-catenin (1:1000; cat. no. $8480 \mathrm{~T})$, anti-cyclin D1 (1:1000; cat. no. $3300 \mathrm{~T})$, and antiGAPDH (1:1000; cat. no. 5174S) antibodies were purchased from Cell Signaling Technology (Boston, MA, USA). AntiWnt (1:1000; cat. no. ab28472) antibody was the product of Abcam company (Cambridge, UK).

2.9. Statistical Analysis. All statistical analyses were conducted using SPSS 14.0 software (Chicago, IL). All experimental results were expressed as mean \pm SD. Statistical comparisons were made by two-tailed Student's $t$ test or oneway analysis of variance (ANOVA). $\mathrm{P}<0.05$ was considered statistically significant.

\section{Results}

3.1. The Expression of SIRT7 Is Suppressed Highly in ox-LDLStimulated HAVSMCs. To determine whether the successful AS cells model was made, the immunofluorescence staining and protein analysis were employed in the present study. Our data indicated that treatment with ox-LDL led to a marked increase in the level of VSMC-specific marker gene $\alpha$-SMA in immunofluorescence imaging [26], which was consistent with the $\alpha$-SMA protein expression level (Figures 1(a) and 1(b)).

3.2. Silencing or Overexpression of SIRT7 Affects Cells Proliferation In ox-LDL-Stimulated HAVSMCs. In our study, the results demonstrated that the protein and mRNA expression levels of SIRT7 were reduced after HAVSMCs underwent ox-LDL stimulation (Figures 1(c) and 1(d)). To test the effects of SIRT7 on cells proliferation, SIRT7 silencing or overexpression was made with siRNA or pcDNA3.1 plasmid successfully, which was shown from the decreasing or increasing protein and mRNA expressions of SIRT7 (Figures $1(\mathrm{e})-1(\mathrm{~h}))$. At the same time, the expression of $\alpha$-SMA was increased in siRNA-SIRT7 group. However, the level of $\alpha$ SMA was decreased after SIRT7 overexpression (Figure 2). In addition, the CCK-8 assay suggested that SIRT7 knockdown promoted HAVSMCs proliferation induced by ox-LDL (Figure 3(a)), while overexpression of SIRT7 had opposite results. Furthermore, cell cycle analysis indicated that the percentage of cells at S phase in siRNA-SIRT7 group was significantly higher than that of siRNA-NC group, while G1 and G2 phases exhibited reverse results, whereas the SIRT7 overexpression exhibited a cycle arrest (Figures 3(b) and 3(c)). The results indicate that SIRT7 inhibition promotes cell proliferation, while SIRT7 overexpression impedes it in ox-LDL-stimulated HAVSMCs.

3.3. Silencing or Overexpression of SIRT7 Affects Cells Migration in ox-LDL-Stimulated HAVSMCs. We monitored cells migration by wound-healing assay. From the results, we found that the relative distance was decreased in SIRT7 knockdown group compared with that in knockdown negative control group at the time of $24 \mathrm{~h}$ after scratch (Figures 4(a) and 4(b)). Importantly, the relative distance was increased after transfection with pcDNA3.1 plasmid. From these results, we find that SIRT7 silencing accelerates cell migration, whereas SIRT7 overexpression retards it in oxLDL-stimulated HAVSMCs.

3.4. SIRT7 Regulates Cells Proliferation and Migration via Wnt/ $\beta$-Catenin Signaling Pathway. To further explore the molecular mechanism underlying the SIRT7-mediated protective effects on ox-LDL-induced proliferation and migration of HAVSMCs, the following study investigated the relative variation in $\mathrm{Wnt} / \beta$-catenin signaling. After treatment with ox-LDL, the protein expression levels of Wnt, $\beta$-catenin, and cyclin D1 were decreased extremely in SIRT7 knockdown HAVSMCs (Figure 5(a)); however, they were increased 
(a)
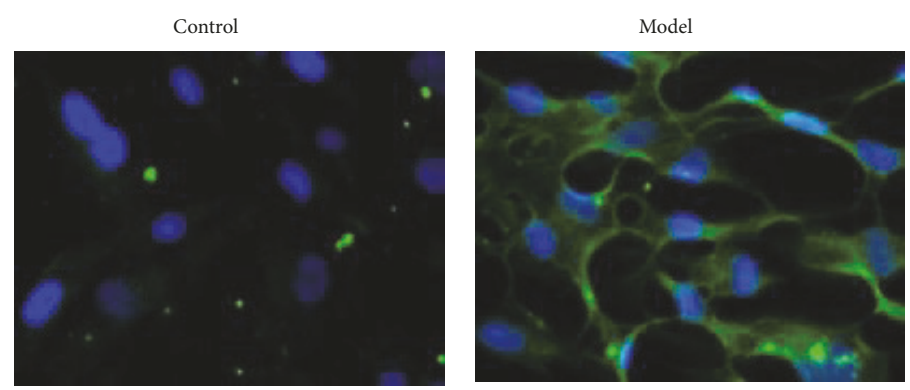

(c)

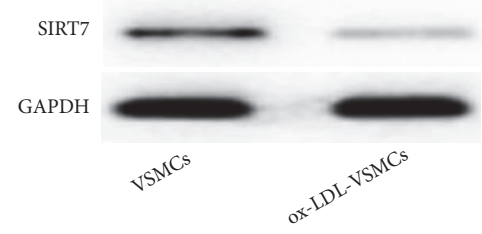

(e)

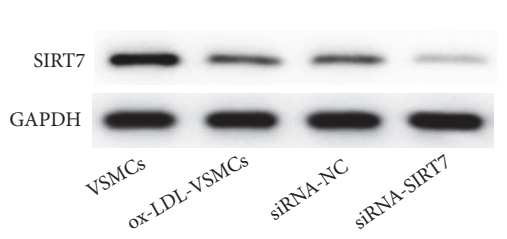

(g)

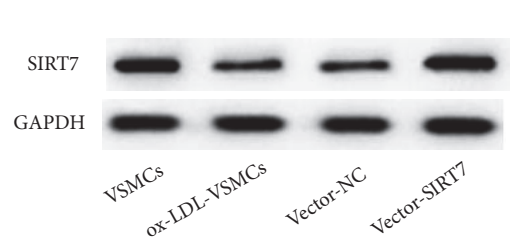

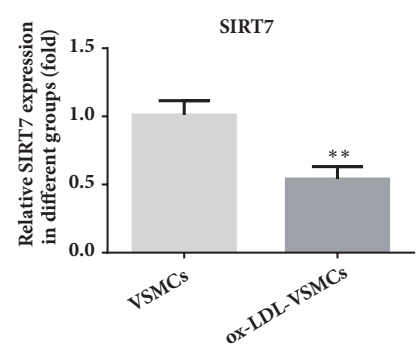

(d)

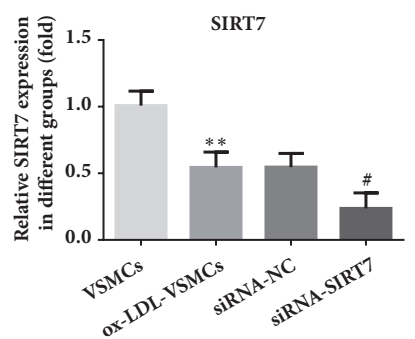

SIRT7

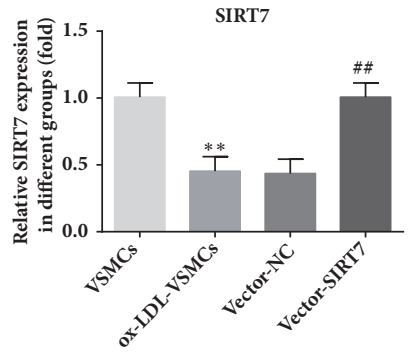

(f)

(h) (b)
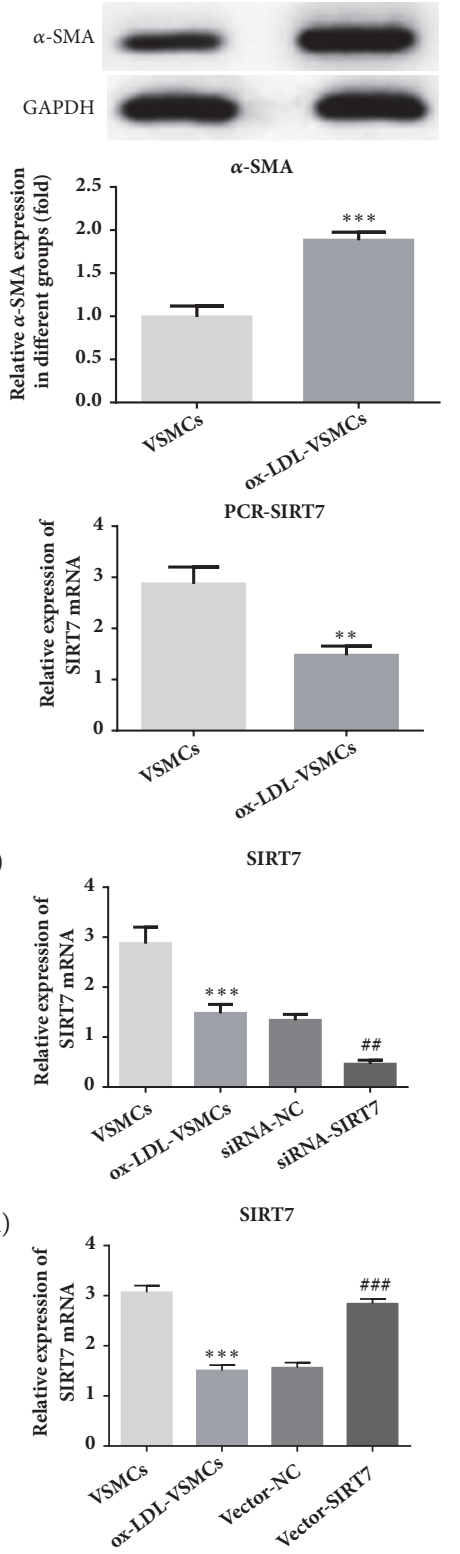

FIgURE 1: The $\alpha$-SMA and SIRT7 expression in ox-LDL-treated HAVSMCs. (a) Immunofluorescence staining was used to detect $\alpha$-SMA in HAVSMCs stimulated with ox-LDL. Nuclei were stained with DAPI (blue), 200× magnification. (b) The protein expression level of $\alpha$-SMA was measured by Western blot. ${ }^{* * *} \mathrm{P}<0.001$ versus VSMCs. The protein level of SIRT7 was measured by Western blot (c) and RT-qPCR (d) in ox-LDL-treated HAVSMCs. ${ }^{* *} \mathrm{P}<0.01$ versus VSMCs. The expression level of SIRT7 was measured by Western blot (e) and RT-qPCR (f) after silencing of SIRT7 in ox-LDL-treated HAVSMCs. ${ }^{* *} \mathrm{P}<0.01$ and ${ }^{* * *} \mathrm{P}<0.001$ versus VSMCs; ${ }^{*} \mathrm{P}<0.05$ and ${ }^{\# \#} \mathrm{P}<0.01$ versus siRNA-NC. The expression level of SIRT7 was measured by Western blot (g) and RT-qPCR (h) after overexpression of SIRT7 in ox-LDL-treated HAVSMCs. ${ }^{* *} \mathrm{P}<0.01$ and ${ }^{* * *} \mathrm{P}<0.001$ versus VSMCs; ${ }^{\# \#} \mathrm{P}<0.01$ and ${ }^{\# \# \#} \mathrm{P}<0.001$ versus Vector-NC.

obviously in SIRT7 overexpression HAVSMCs (Figure 5(b)). Furthermore, DKK-1, a secretory glycoprotein, was used to block the Wnt/ $\beta$-catenin signaling pathway in our study. The results indicated that the abilities of cells proliferation and migration were augmented after DKK-1 was employed in SIRT7 overexpression HAVSMCs, which were exhibited in Figures 3 and 4 . These results uncover that SIRT7 regulates cells proliferation and migration via $\mathrm{Wnt} / \beta$-catenin signaling pathway.

\section{Discussion}

It is well known that AS is a chronic degenerative disease and has become a predominant factor of a plenty of cardiovascular diseases, which has high morbidity and mortality. However, the specific pathogenesis mechanisms of AS are still not fully elucidated. In this study, we found that SIRT7 regulates the VSMCs proliferation and migration induced by ox-LDL via Wnt/ $\beta$-catenin signaling pathway. 


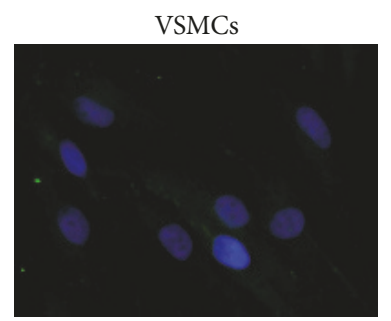

VSMCs
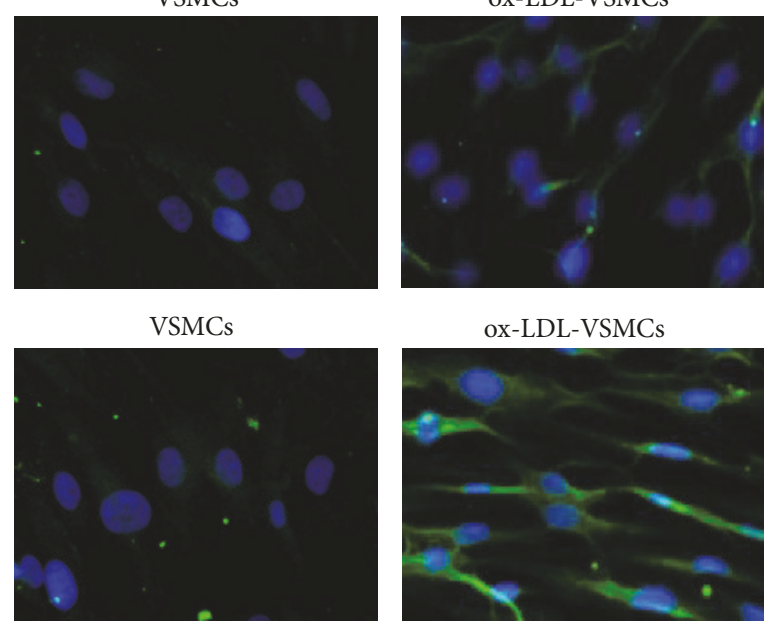

ox-LDL-VSMCs

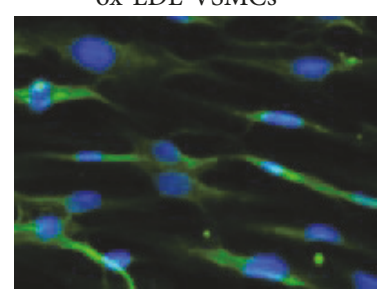

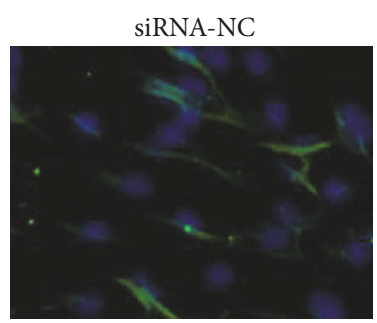

Vector-NC

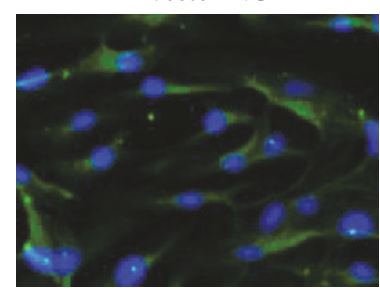

(a)

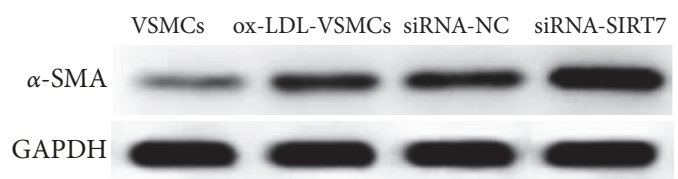

$\alpha$-SMA

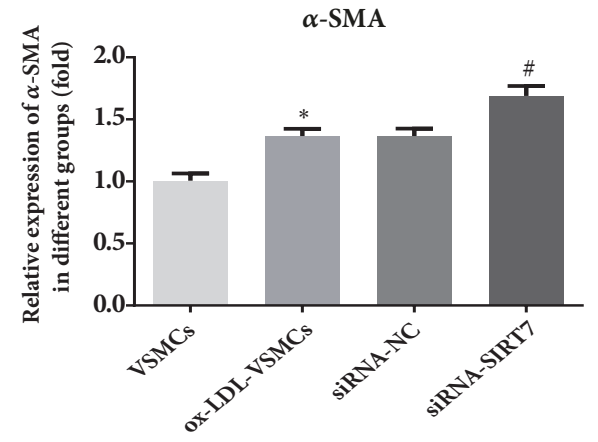

(b)
VSMCs ox-LDL-VSMCs Vector-NC Vector-SIRT7
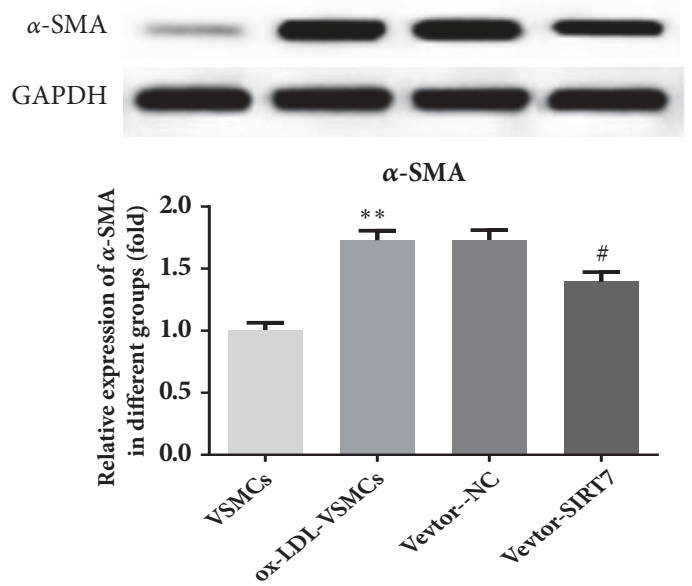

(c)

FIGURE 2: (a) The level of $\alpha$-SMA after SIRT7 silencing or overexpression in ox-LDL-treated HAVSMCs was measured by immunofluorescence staining. Nuclei were stained with DAPI (blue), 200× magnification. (b) The protein expression level of $\alpha$-SMA after SIRT7 silencing (b) or overexpression (c) in ox-LDL-treated HAVSMCs was assessed by Western blot. ${ }^{*} \mathrm{P}<0.05$ and ${ }^{* *} \mathrm{P}<0.01$ versus VSMCs; ${ }^{*} \mathrm{P}<0.05$ versus siRNA-NC or Vector-NC.

Aberrantly proliferation and migration of VSMCs play a considerable role in the progression of AS [27]. It has been well documented that ox-LDL exerts a promotion effect on cellular proliferation and migration [28]. Evidence indicates that SITR7 as an important cellular regulator plays critical roles in cell proliferation, migration, survival, and protein synthesis [11-13]. Furthermore, SIRT7 possesses antiproliferative effects in the control of cellular growth and proliferation $[12,29]$, which suggests that SIRT7 may inhibit the development procession of AS. Decreased expression level of SIRT7 was detected in ox-LDL-treated HAVSMCs in our study. Additionally, silencing of SIRT7 by siRNA considerably accelerated cellular proliferation, coupled with the increasing percentage of VSMCs at $S$ phase and decreasing that of G1 and G2 phases detected by cell cycle analysis. At the same time, cellular migration was expedited assessed by the woundhealing assay. Moreover, overexpression of SIRT7 exhibited an inhibition effect on HAVSMCs proliferation and migration in our study. To the best of our knowledge, the results indicate that SIRT7 plays a protective role in regulating cellular proliferation and migration in ox-LDL-induced HAVSMCs.

The Wnt/ $\beta$-catenin signaling is an evolutionarily conserved pathway and activation of this signaling regulates the expression of downstream target genes including cyclin D1, subsequently resulting in many variations of pathophysiological behaviors, such as cell differentiation, proliferation, migration, and polarity $[30,31]$. Accumulating evidence indicated that Wnt $/ \beta$-catenin signaling has been shown to be involved in SMA and calponin expression which are marker genes during the progression of AS [32, 33]. In addition, it has been reported that SIRT7 is essential for osteogenic differentiation of bone marrow stem cells, partly by activation of the Wnt $/ \beta$-catenin signaling pathway [18]. To investigate the potential mechanisms of SIRT7-mediated protective 


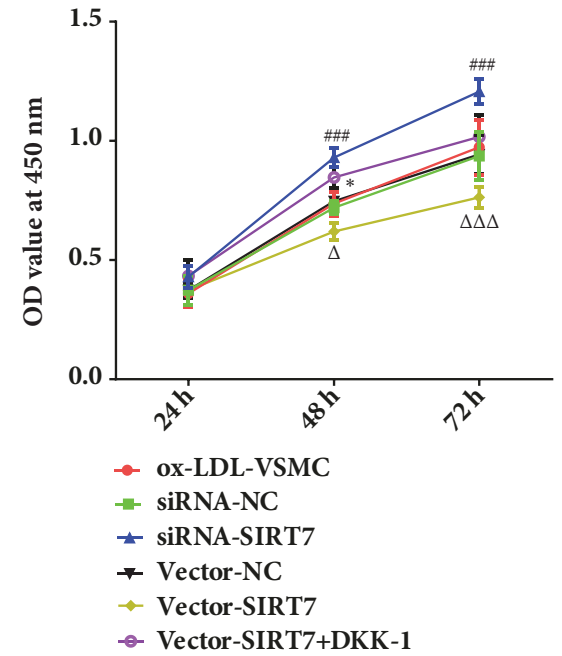

(a)

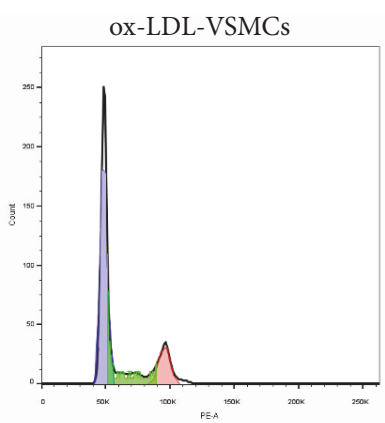

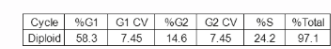

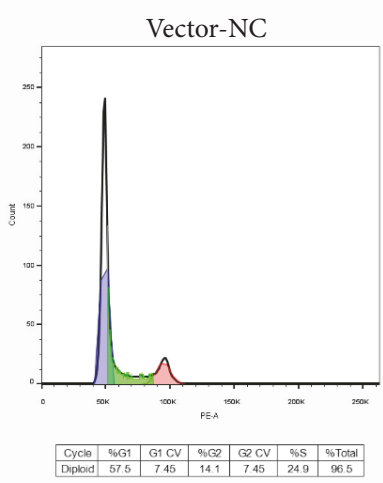

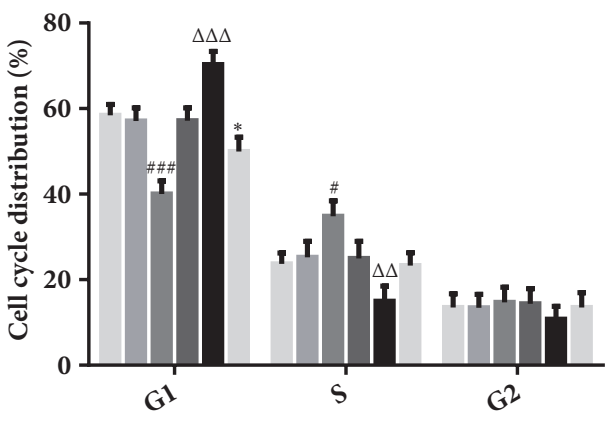

ox-LDL-VSMC

SiRNA-NC

siRNA-SIRT7

Vector-NC

Vector-SIRT7

Vector-SIRT7+DKK-1

(b)
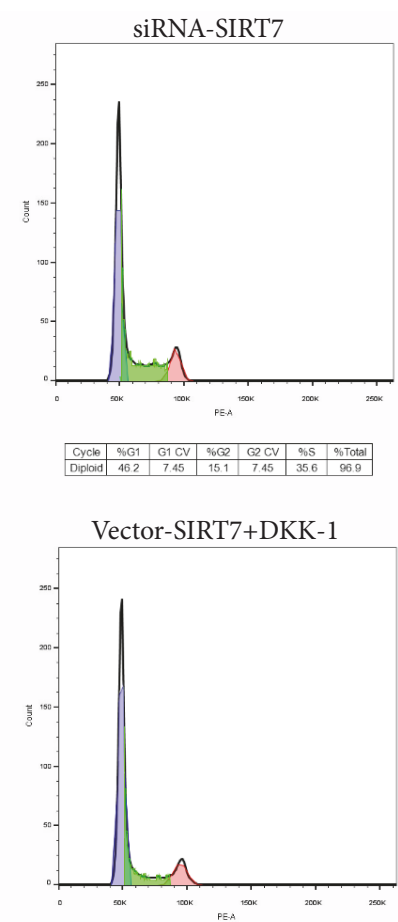

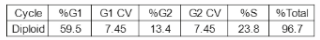

(c)

FIGURE 3: The effects of SIRT7 knockdown, overexpression, or treatment with DKK-1 on HAVSMCs proliferation stimulated by ox-LDL. (a) CCK-8 cell proliferation assay. ${ }^{\# \# \# ~} \mathrm{P}<0.001$ versus siRNA-NC; ${ }^{\triangle} \mathrm{P}<0.05$ and ${ }^{\triangle \triangle \triangle} \mathrm{P}<0.001$ versus Vector-NC; ${ }^{*} \mathrm{P}<0.05$ versus Vector-SIRT7. (b) Cell cycle distribution (\%) in G1, S, and G2 phases. ${ }^{\#} \mathrm{P}<0.05$ and ${ }^{\# \#} \mathrm{P}<0.001$ versus siRNA-NC; ${ }^{\triangle \Delta} \mathrm{P}<0.01$ and ${ }^{\triangle \triangle \triangle} \mathrm{P}<0.001$ versus Vector-NC; ${ }^{*} \mathrm{P}<0.05$ versus Vector-SIRT7. (c) Cell cycle analysis.

effects on ox-LDL-induced proliferation and migration of HAVSMCs, Wnt/ $\beta$-catenin signaling intrigues our interest because of its crucial role in regulating essential aspects of cell proliferation and migration $[34,35]$. The present study aimed to evaluate whether Wnt $/ \beta$-catenin signaling was affected by the knockdown or overexpression of SIRT7 in HAVSMCs.
In our present research, we found that the expression levels of $\beta$-catenin and cyclin D1 were notably decreased in SIRT7 silencing HAVSMCs, while an increased result was detected in SIRT7 overexpression condition. Taken together, these findings indicated that there was a regulatory relationship between SIRT7 and Wnt/ $\beta$-catenin signaling in HAVSMCs. 
ox-LDL-VSMCs
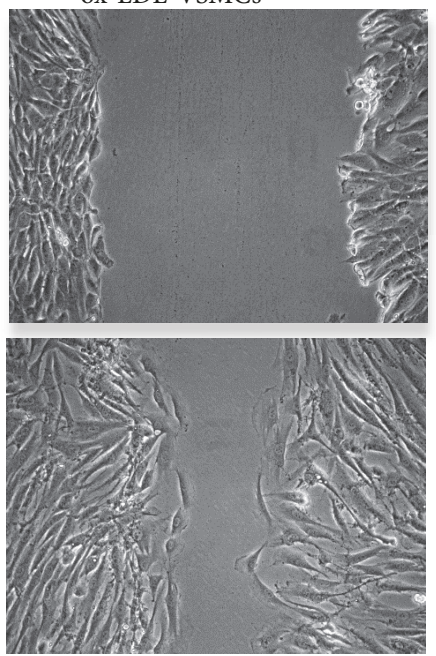

Vector-NC

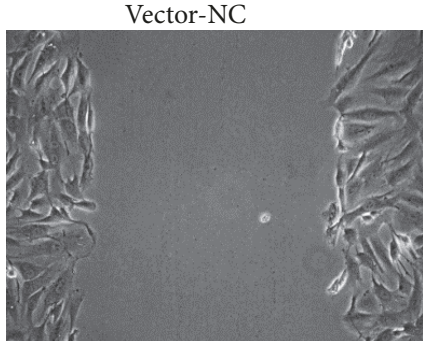

$0 \mathrm{~h}$

$24 \mathrm{~h}$

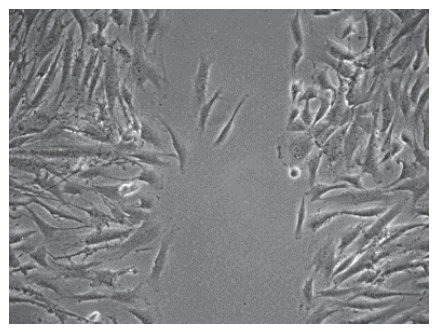

siRNA-NC
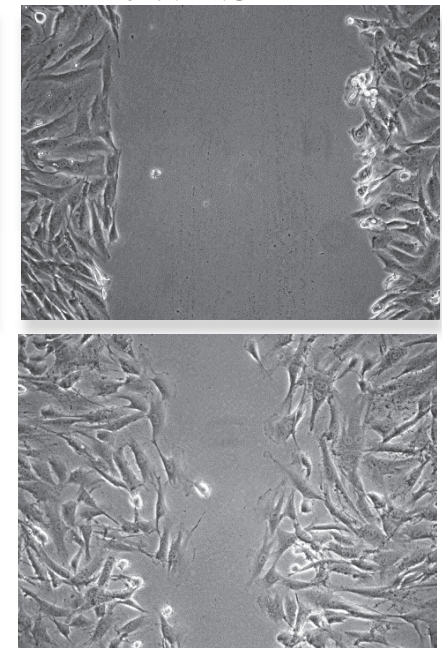

Vector-SIRT7
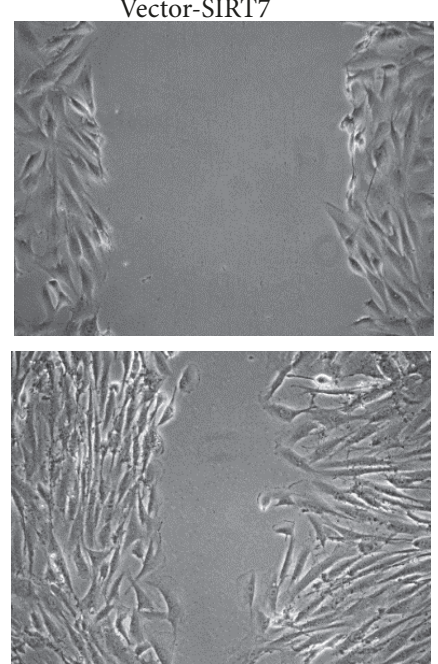

(a)
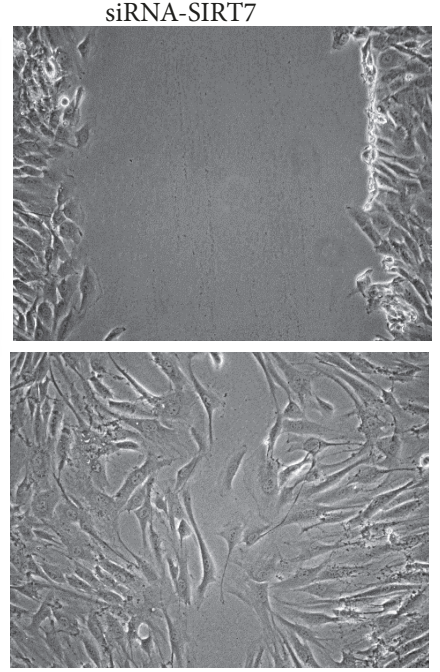

Vector-SIRT7+DKK-1
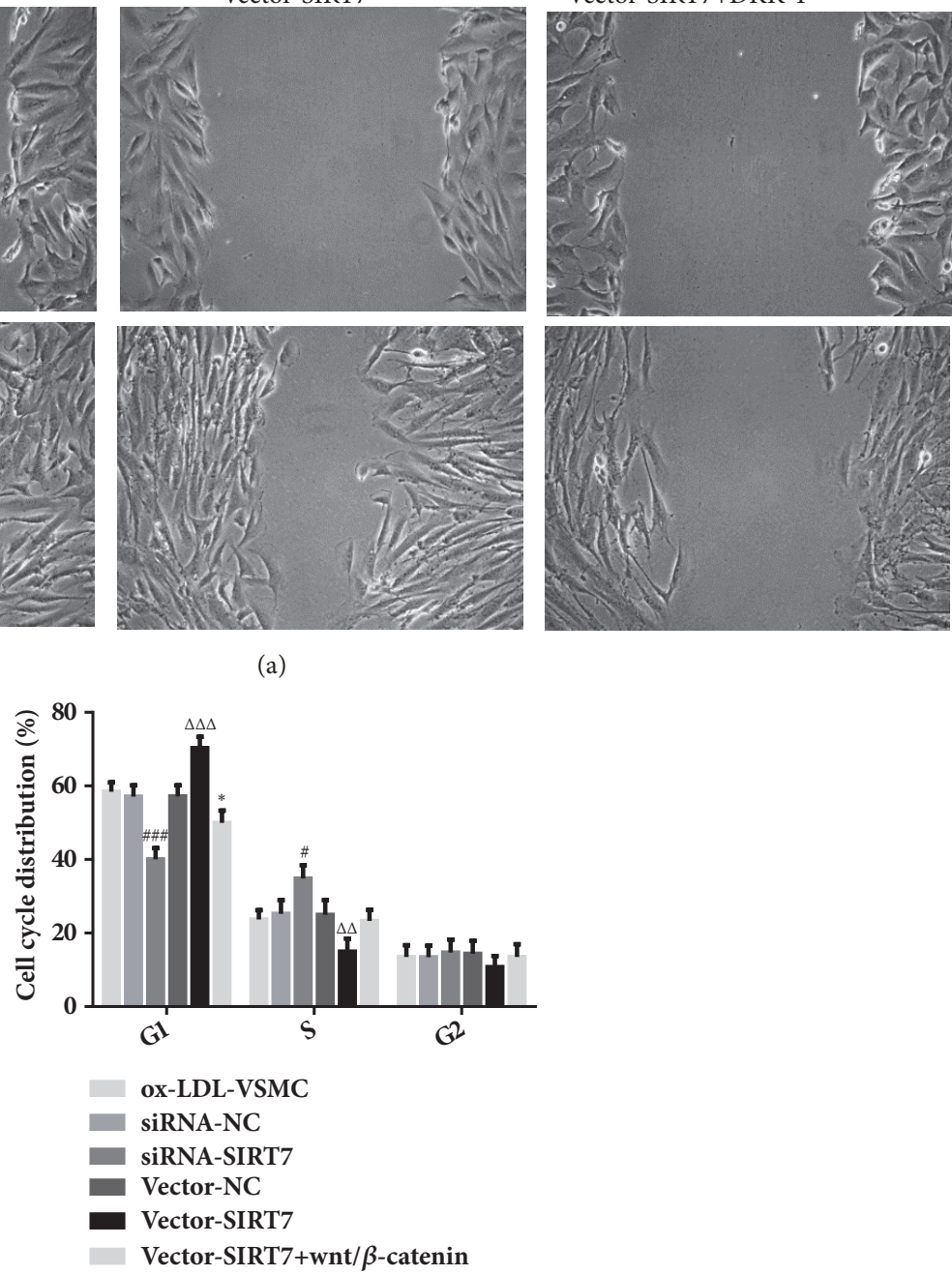

(b)

FIGURE 4: The effects of SIRT7 knockdown, overexpression, or treatment with DKK-1 on HAVSMCs migration stimulated by ox-LDL. (a) Images of the migration of HAVSMCs at 0 and $24 \mathrm{~h}$ ( $4 \times$ magnification). (b) The wound closure of ox-LDL treatment groups at 0 and $24 \mathrm{~h}$. ${ }^{\# \# \#} \mathrm{P}<0.001$ versus siRNA-NC; ${ }^{\triangle \triangle} \mathrm{P}<0.001$ versus Vector-NC; ${ }^{* *} \mathrm{P}<0.01$ versus Vector-SIRT7. 

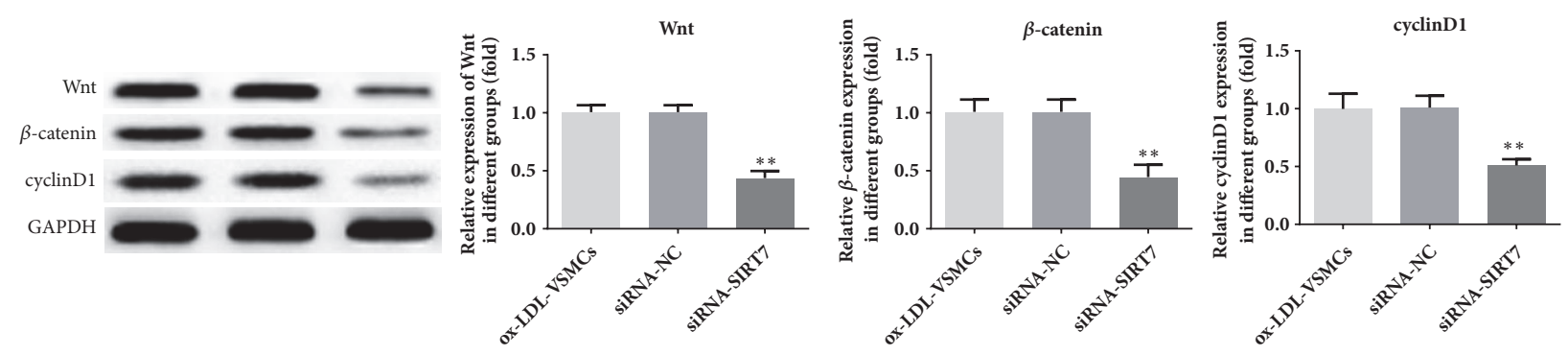

(a)
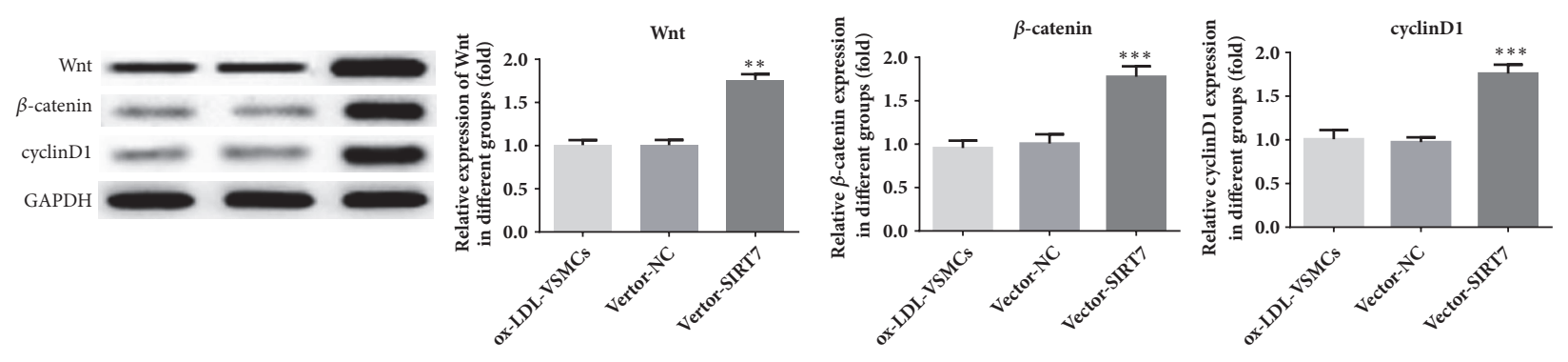

(b)

Figure 5: The protein expression levels of Wnt, $\beta$-catenin, and cyclin D1. (a) The protein expression levels of Wnt, $\beta$-catenin, and cyclin D1 in SIRT7 knockdown HAVSMCs. ${ }^{* *} \mathrm{P}<0.01$ and ${ }^{* * *} \mathrm{P}<0.001$ versus ox-LDL-VSMC; ${ }^{\# \#} \mathrm{P}<0.01$ and ${ }^{\# \# \#} \mathrm{P}<0.001$ versus siRNA-NC. (b) The protein expression levels of Wnt, $\beta$-catenin, and cyclin D1 in SIRT7 overexpression on HAVSMCs. ${ }^{* * *} \mathrm{P}<0.001$ versus ox-LDL-VSMC; ${ }^{\# \# \# ~} \mathrm{P}$ $<0.001$ versus Vector-NC.

To further elucidate the underlying regulation between SIRT7 and Wnt/ $\beta$-catenin signaling, DKK-1, a classical inhibitor of canonical Wnt/ $\beta$-catenin signaling, was employed in our study [36]. The results indicated that the levels of HAVSMCs proliferation and migration induced by ox-LDL were remarkably decreased assessed by CKK-8, cytometric analysis, and wound-healing assay, respectively, which verified our hypothesis and further suggested that SIRT7 regulates the VSMCs proliferation and migration in AS via $\mathrm{Wnt} / \beta$-catenin signaling pathway.

\section{Conclusion}

According to our results, the present study demonstrated that SIRT7 was significantly downregulated in ox-LDL-stimulated HAVSMCs. SIRT7 knockdown notably promoted cell proliferation and migration and downregulated the expression of $\beta$-catenin and cyclin D1, while SIRT7 overexpression had reverse results. These findings indicated that SIRT7 plays a protective role in regulating proliferation and migration of AS via $\mathrm{Wnt} / \beta$-catenin signaling pathways.

\section{Data Availability}

The data used to support the findings of this study are included within the article.

\section{Conflicts of Interest}

The authors declare that there are no conflicts of interest.

\section{Acknowledgments}

The present study was supported by Sichuan provincial health office fund (10035).

\section{References}

[1] K. Chen and S. H. Juo, "MicroRNAs in atherosclerosis," Kaohsiung Journal of Medical Sciences, vol. 28, no. 12, pp. 631-640, 2012.

[2] A. C. Doran, N. Meller, and C. A. McNamara, "Role of smooth muscle cells in the initiation and early progression of atherosclerosis," Arteriosclerosis, Thrombosis, and Vascular Biology, vol. 28, no. 5, pp. 812-819, 2008.

[3] P. Wang, T.-Y. Xu, Y.-F. Guan et al., "Vascular smooth muscle cell apoptosis is an early trigger for hypothyroid atherosclerosis," Cardiovascular Research, vol. 102, no. 3, pp. 448-459, 2014.

[4] A. Pirillo, G. D. Norata, and A. L. Catapano, "LOX-1, OxLDL, and atherosclerosis," Mediators of Inflammation, vol. 2013, Article ID 152786, 12 pages, 2013.

[5] L. Tian, K. Chen, J. Cao et al., "Galectin-3-induced oxidized low-density lipoprotein promotes the phenotypic transformation of vascular smooth muscle cells," Molecular Medicine Reports, vol. 12, no. 4, pp. 4995-5002, 2015.

[6] R. H. Houtkooper, E. Pirinen, and J. Auwerx, "Sirtuins as regulators of metabolism and healthspan," Nature Reviews Molecular Cell Biology, vol. 13, no. 4, pp. 225-238, 2012.

[7] L. Bosch-Presegué and A. Vaquero, "The dual role of sirtuins in cancer," Genes \& Cancer, vol. 2, no. 6, pp. 648-662, 2011.

[8] L. Yang, J. Zhang, C. Yan et al., "SIRT1 regulates CD40 expression induced by TNF- $\alpha$ via NF- $\kappa$ B pathway in endothelial 
cells," Cellular Physiology and Biochemistry, vol. 30, no. 5, pp. 1287-1298, 2012.

[9] I. Gorenne, S. Kumar, K. Gray et al., "Vascular smooth muscle cell sirtuin 1 protects against dna damage and inhibits atherosclerosis," Circulation, vol. 127, no. 3, pp. 386-396, 2013.

[10] M.-J. Zhang, Y. Zhou, L. Chen et al., "Impaired SIRT1 promotes the migration of vascular smooth muscle cell-derived foam cells," Histochemistry and Cell Biology, vol. 146, no. 1, pp. 33-43, 2016.

[11] S. Kiran, T. Anwar, M. Kiran, and G. Ramakrishna, "Sirtuin 7 in cell proliferation, stress and disease: Rise of the Seventh Sirtuin!," Cellular Signalling, vol. 27, no. 3, pp. 673-682, 2015.

[12] E. Ford, R. Voit, G. Liszt, C. Magin, I. Grummt, and L. Guarente, "Mammalian Sir2 homolog SIRT7 is an activator of RNA polymerase I transcription," Genes \& Development, vol. 20, no. 9, pp. 1075-1080, 2006.

[13] M. F. Barber, E. Michishita-Kioi, Y. Xi et al., "SIRT7 links H3K18 deacetylation to maintenance of oncogenic transformation," Nature, vol. 486, no. 7405, pp. 114-118, 2012.

[14] S. Zhang, P. Chen, Z. Huang et al., "Sirt7 promotes gastric cancer growth and inhibits apoptosis by epigenetically inhibiting miR-34a," Scientific Reports, vol. 5, article 9787, 2015.

[15] K.-L. Chen, L. Li, F.-X. Yang, C.-M. Li, Y.-R. Wang, and G.-L. Wang, "SIRT7 depletion inhibits cell proliferation, migration, and increases drug sensitivity by activating p38MAPK in breast cancer cells," Journal of Cellular Physiology, 2018.

[16] W. Li, D. Zhu, and S. Qin, "SIRT7 suppresses the epithelialto-mesenchymal transition in oral squamous cell carcinoma metastasis by promoting SMAD4 deacetylation," Journal of Experimental \& Clinical Cancer Research, vol. 37, no. 1, 2018.

[17] Z. Chen, X. Chen, W. Han et al., "The expression of SIRT7 was down-regulated and correlated with tumor size in thyroid carcinoma," International Journal of Clinical and Experimental Medicine, vol. 9, p. 5, 2016.

[18] E. E. Chen, W. Zhang, C. C. Ye et al., "Knockdown of SIRT7 enhances the osteogenic differentiation of human bone marrow mesenchymal stem cells partly via activation of the $\mathrm{Wnt} / \beta$ catenin signaling pathway," Cell Death \& Disease, vol. 8, no. 9, p. e3042, 2017.

[19] H. Quasnichka, S. C. Slater, C. A. Beeching, M. Boehm, G. B. Sala-Newby, and S. J. George, "Regulation of Smooth Muscle Cell Proliferation by $\beta$-Catenin/T-Cell Factor Signaling Involves Modulation of Cyclin D1 and p21 Expression," Circulation Research, vol. 99, no. 12, pp. 1329-1337, 2006.

[20] J. Shao, S. Cheng, J. M. Pingsterhaus, N. Charlton-Kachigian, A. P. Loewy, and D. A. Towler, "Msx2 promotes cardiovascular calcification by activating paracrine Wnt signals," The Journal of Clinical Investigation, vol. 115, no. 5, pp. 1210-1220, 2005.

[21] K. M. Cadigan and R. Nusse, "Wnt signaling: a common theme in animal development," Genes \& Development, vol. 11, no. 24, pp. 3286-3305, 1997.

[22] A. Bedel, A. Nègre-Salvayre, S. Heeneman et al., "ECadherin/ $\beta$-Catenin/T-Cell Factor Pathway Is Involved in Smooth Muscle Cell Proliferation Elicited by Oxidized LowDensity Lipoprotein," Circulation Research, vol. 103, no. 7, pp. 694-701, 2008.

[23] K. Schaale, J. Neumann, D. Schneider, S. Ehlers, and N. Reiling, "Wnt signaling in macrophages: Augmenting and inhibiting mycobacteria-induced inflammatory responses," European Journal of Cell Biology, vol. 90, no. 6-7, pp. 553-559, 2011.
[24] I. Gomceli, E. B. Bostanci, I. Ozer et al., "A novel screening biomarker in gastric cancer: Serum Dickkopf-1," HepatoGastroenterology, vol. 59, no. 117, pp. 1661-1664, 2012.

[25] K. J. Livak and T. D. Schmittgen, "Analysis of relative gene expression data using real-time quantitative PCR and the $2^{-\Delta \Delta C_{T}}$ method," Methods, vol. 25, no. 4, pp. 402-408, 2001.

[26] J. K. Salabei, T. D. Cummins, M. Singh, S. P. Jones, A. Bhatnagar, and B. G. Hill, "PDGF-mediated autophagy regulates vascular smooth muscle cell phenotype and resistance to oxidative stress," Biochemical Journal, vol. 451, no. 3, pp. 375-388, 2013.

[27] V. J. Dzau, R. C. Braun-Dullaeus, and D. G. Sedding, "Vascular proliferation and atherosclerosis: new perspectives and therapeutic strategies," Nature Medicine, vol. 8, no. 11, pp. 1249-1256, 2002.

[28] Z. Ding, S. Liu, B. Yang, Y. Fan, and X. Deng, "Effect of oxidized low-density lipoprotein concentration polarization on human smooth muscle cells' proliferation, cycle, apoptosis and oxidized low-density lipoprotein uptake," Journal of the Royal Society Interface, vol. 9, no. 71, pp. 1233-1240, 2012.

[29] O. Vakhrusheva, D. Braeuer, Z. Liu, T. Braun, and E. Bober, "Sirt7-dependent inhibition of cell growth and proliferation might be instrumental to mediate tissue integrity during aging," Journal of Physiology and Pharmacology, vol. 59, no. 9, pp. 201212, 2008.

[30] R. Nusse, "Wnt signaling," Cold Spring Harbor Perspectives in Biology, vol. 4, no. 5, p. 11, 2012.

[31] F. Wang, Z. Liu, S. Park et al., "Myeloid $\beta$-Catenin Deficiency Exacerbates Atherosclerosis in Low-Density Lipoprotein Receptor-Deficient Mice," Arteriosclerosis, Thrombosis, and Vascular Biology, vol. 38, no. 7, pp. 1468-1478, 2018.

[32] S. Menini, C. Iacobini, C. Ricci et al., "The galectin-3/RAGE dyad modulates vascular osteogenesis in atherosclerosis," Cardiovascular Research, vol. 100, no. 3, pp. 472-480, 2013.

[33] J. M. Carthy, Z. Luo, and B. M. McManus, "WNT3A induces a contractile and secretory phenotype in cultured vascular smooth muscle cells that is associated with increased gap junction communication," Laboratory Investigation, vol. 92, no. 2, pp. 246-255, 2012.

[34] D. Xie, D. Yin, X. Tong et al., "Cyr61 Is Overexpressed in Gliomas and Involved in Integrin-Linked Kinase-Mediated Akt and $\beta$-Catenin-TCF/Lef Signaling Pathways," Cancer Research, vol. 64, no. 6, pp. 1987-1996, 2004.

[35] K. B. Lee, S. Ye, M. H. Park, B. H. Park, J. Lee, and S. M. Kim, "p63-Mediated activation of the $\beta$-catenin/c-Myc signaling pathway stimulates esophageal squamous carcinoma cell invasion and metastasis," Cancer Letters, vol. 353, no. 1, pp. 124-132, 2014.

[36] C. A. Gregory, H. Singh, A. S. Perry, and D. J. Prockop, "The Wnt signaling inhibitor Dickkopf-1 is required for reentry into the cell cycle of human adult stem cells from bone marrow," The Journal of Biological Chemistry, vol. 278, no. 30, pp. 2806728078, 2003. 


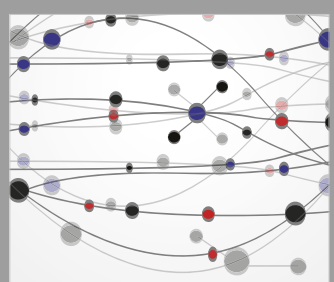

The Scientific World Journal
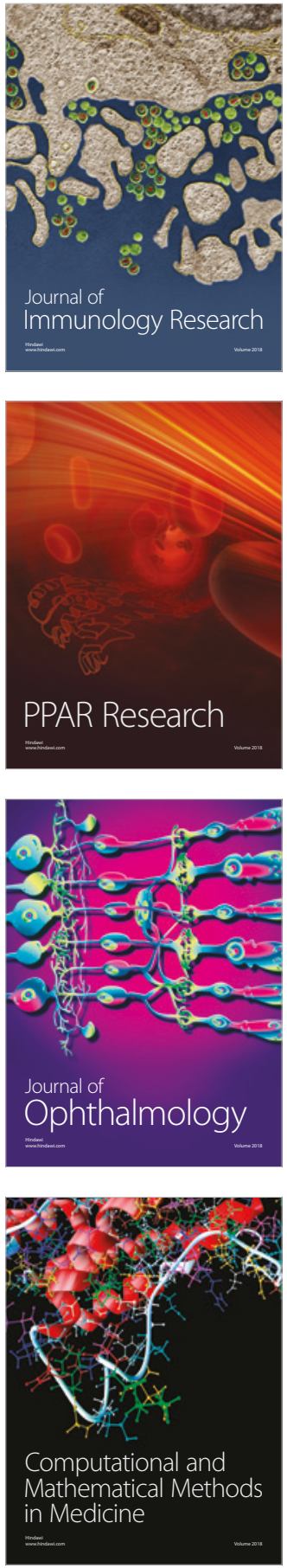

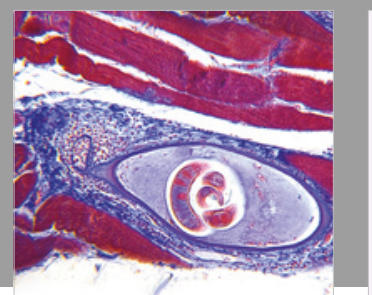

Gastroenterology Research and Practice

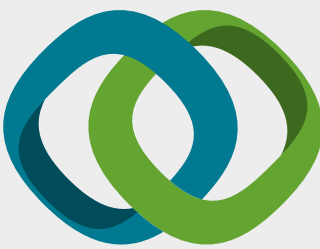

\section{Hindawi}

Submit your manuscripts at

www.hindawi.com
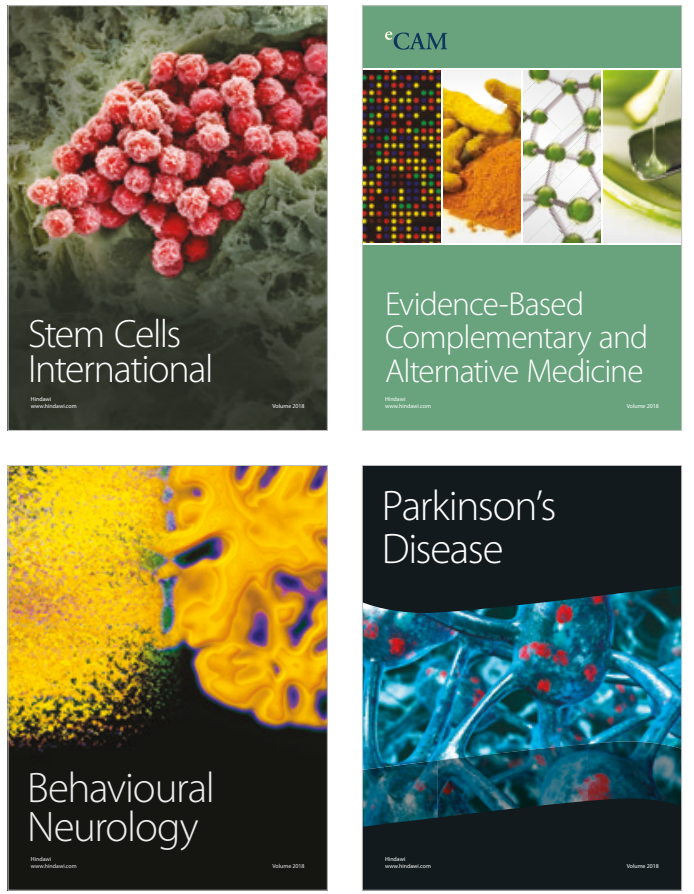

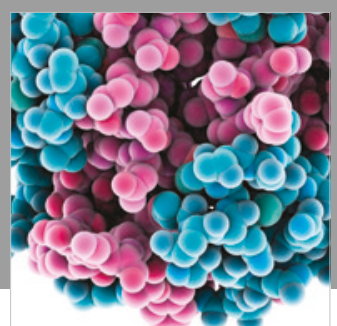

ournal of

Diabetes Research

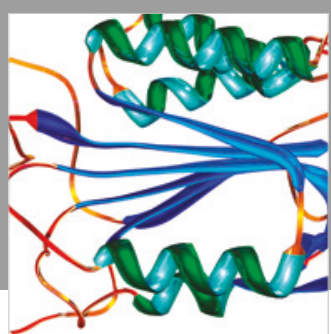

Disease Markers
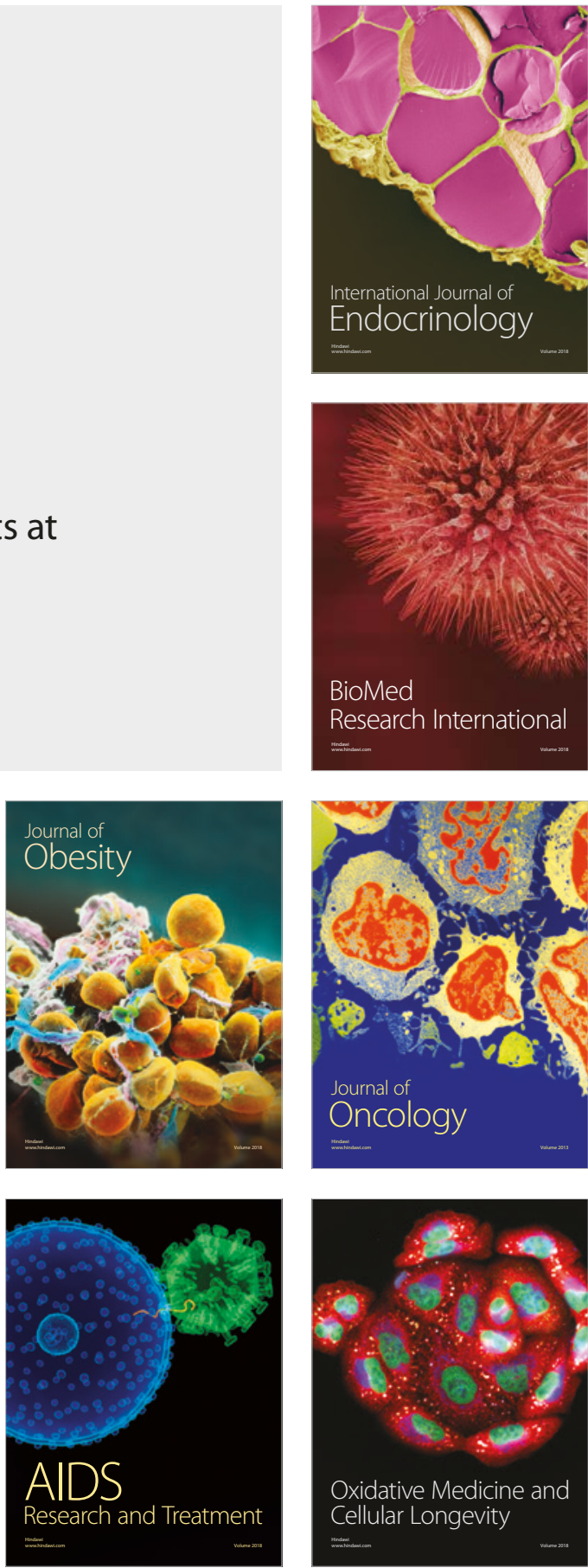